\title{
Guided Internet-based vs. face-to-face cognitive behavior therapy for psychiatric and somatic disorders: a systematic review and meta-analysis
}

\author{
Gerhard Andersson ${ }^{1,2}$, Pim Cuijpers ${ }^{3}$, Per Carlbring ${ }^{4}$, Heleen Riper ${ }^{3,5}$, Erik Hedman ${ }^{6}$ \\ ${ }^{1}$ Department of Behavioural Sciences and Learning, Swedish Institute for Disability Research, University of Linköping, Linköping, Sweden; ${ }^{2}$ Department of Clini- \\ cal Neuroscience, Division of Psychiatry, Karolinska Institutet, Stockholm, Sweden; ${ }^{3}$ Department of Clinical Psychology, VU University Amsterdam, Amsterdam, \\ The Netherlands; ${ }^{4}$ Department of Psychology, University of Stockholm, Stockholm, Sweden; ${ }^{5}$ Leuphana University, Lünebrug, Germany; ${ }^{6}$ Department of Clinical \\ Neuroscience, Osher Center for Integrative Medicine and Division of Psychology, Karolinska Institutet, Stockholm, Sweden
}

Internet-delivered cognitive behavior therapy (ICBT) has been tested in many research trials, but to a lesser extent directly compared to faceto-face delivered cognitive behavior therapy (CBT). We conducted a systematic review and meta-analysis of trials in which guided ICBT was directly compared to face-to-face CBT. Studies on psychiatric and somatic conditions were included. Systematic searches resulted in 13 studies (total $N=1053)$ that met all criteria and were included in the review. There were three studies on social anxiety disorder, three on panic disorder, two on depressive symptoms, two on body dissatisfaction, one on tinnitus, one on male sexual dysfunction, and one on spider phobia. Face-to-face CBT was either in the individual format $(n=6)$ or in the group format $(n=7)$. We also assessed quality and risk of bias. Results showed a pooled effect size (Hedges' g) at post-treatment of -0.01 (95\% CI: -0.13 to 0.12), indicating that guided ICBT and face-to-face treatment produce equivalent overall effects. Study quality did not affect outcomes. While the overall results indicate equivalence, there are still few studies for each psychiatric and somatic condition and many conditions for which guided ICBT has not been compared to face-to-face treatment. Thus, more research is needed to establish equivalence of the two treatment formats.

Key words: Guided Internet-delivered cognitive behavior therapy, face-to-face therapy, anxiety and mood disorders, somatic disorders, metaanalysis

(World Psychiatry 2014;13:288-295)

Internet-delivered psychological treatments have a relatively short history, with the first trials being conducted in late 1990s (1). A large number of programs have been developed, and trials have been conducted for a range of psychiatric and somatic conditions, mostly using Internet-delivered cognitive behavior therapy (ICBT) (2).

Many ICBT programs involve therapist guidance over encrypted e-mail, which to date, with a few exceptions (3), tend to generate larger effects than unguided programs (4). Many interventions are text-based and can be described as online bibliotherapy, even if streamed video clips, audio files and interactive elements are involved. These programs are typically comprised of 6-15 modules, which are text chapters corresponding to sessions in face-to-face therapy, and require little therapist involvement more than guidance and feedback on homework assignments (approximately 10-15 min per client and week). Other programs, such as Interapy (5), require more therapist input, as more text is exchanged between the therapist and the client. Finally, there are real time chat-based Internet treatments in which no therapist time is saved (6).

In terms of content, programs vary as well, but many tend to mirror face-to-face treatments in terms of content and length. Thus, for example, a program for depression can be 10 weeks long, with weekly modules mirroring sessions in manualized cognitive behavior therapy (CBT) (4), and the content may include psychoeducation, behavioral activation, cognitive restructuring, relapse prevention and homework assignments (7).
While most studies have been on ICBT (8), there are also studies on other psychotherapeutic orientations, such as psychodynamic psychotherapy (9), and physical exercise (10). Different forms of ICBT have also been used, such as attention bias modification (11), problem solving therapy (12), and acceptance and commitment therapy (13). In addition to short-term effects indicating equivalence compared to therapist administered therapy (14-16), there are also a few long-term follow-up studies showing lasting effects over as much as 5 years post-treatment (17).

In spite of promising results in the controlled trials, an outstanding question is how well-guided ICBT compares against standard manualized face-to-face treatments. This was partly investigated in a meta-analysis by Cuijpers et al (18), who studied the effects of guided self-help on depression and anxiety vs. face-to-face psychotherapies. They included 21 studies with 810 participants and found no differences between the formats (Cohen's $d=0.02)$. However, that meta-analysis mixed bibliotherapy and Internet interventions and focused on anxiety and depression only. Furthermore, new studies have emerged since that publication. Thus, there is a need for a systematic review and meta-analysis focusing on guided ICBT.

The aim of this study was to investigate the efficacy of guided ICBT compared to face-to-face CBT for psychiatric and somatic disorders. We conducted a systematic review and meta-analysis of studies directly comparing the two treatment formats in randomized trials. We hypothesized that guided ICBT and face-to-face CBT would produce equivalent treatment effects. 


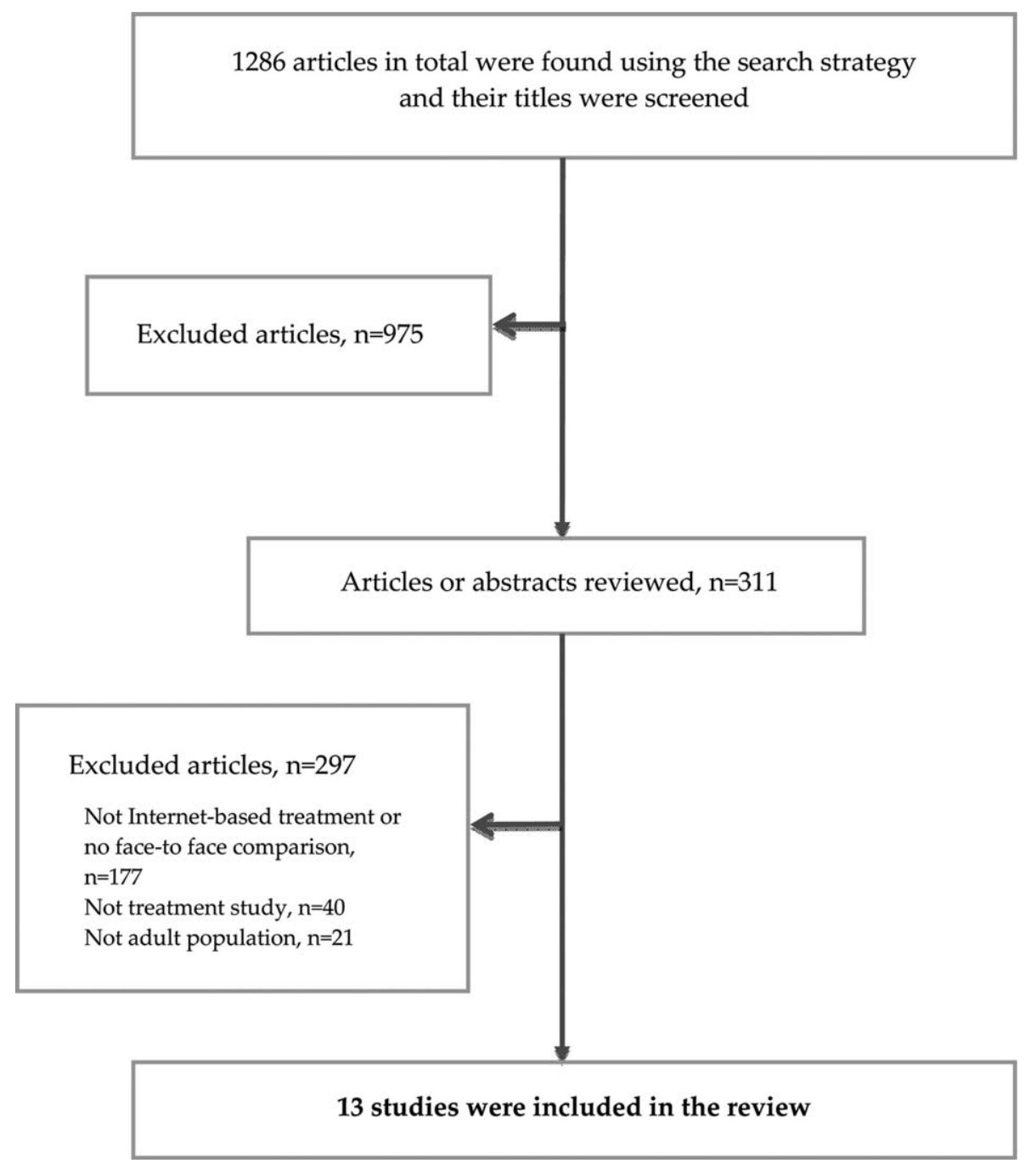

Figure 1 Study inclusion process

\section{METHODS}

This was a systematic review and meta-analysis of original articles investigating the effect of guided ICBT compared to face-to-face treatments. To be included in the review, the original studies had to: a) compare therapistguided ICBT to face-to-face treatment using a randomized controlled design; b) use interventions that were aimed at treatment of psychiatric or somatic disorders (not, for example, prevention or merely psychoeducation); c) compare treatments that were similar in content in both treatment conditions; d) investigate a form of ICBT where the Internet treatment was the main component and not a secondary complement to other therapies; e) investigate a form of full length face-to-face treatment; f) report outcome data from an adult patient sample; g) report outcomes in terms of assessment of symptoms of the target problem; and h) be written in English. We included only studies in which there was some therapist contact during the trial (7).
We calculated effect sizes based on the primary outcome measure at post-treatment in each study. If no primary outcome measure was specified in the original study, a validated measure assessing target symptoms of the clinical problem was used, following the procedures by Thomson and Page (19).

To identify studies, systematic searches in PubMed (Medline database) were conducted using various search terms related to psychiatric and somatic disorders, such as "depression", "panic disorder", "social phobia", "social anxiety disorder", "generalized anxiety disorder", "obsessivecompulsive disorder", "post-traumatic stress disorder", "specific phobia", "hypochondriasis", "bulimia", "tinnitus", "erectile dysfunction", "chronic pain", or "fatigue". These search terms relating to the clinical problem were combined with "Internet" or "computer", or "computerized", and the search filter "randomized controlled trial" was used.

In addition to the above, reference lists in the included studies were checked for potential additional studies. We 


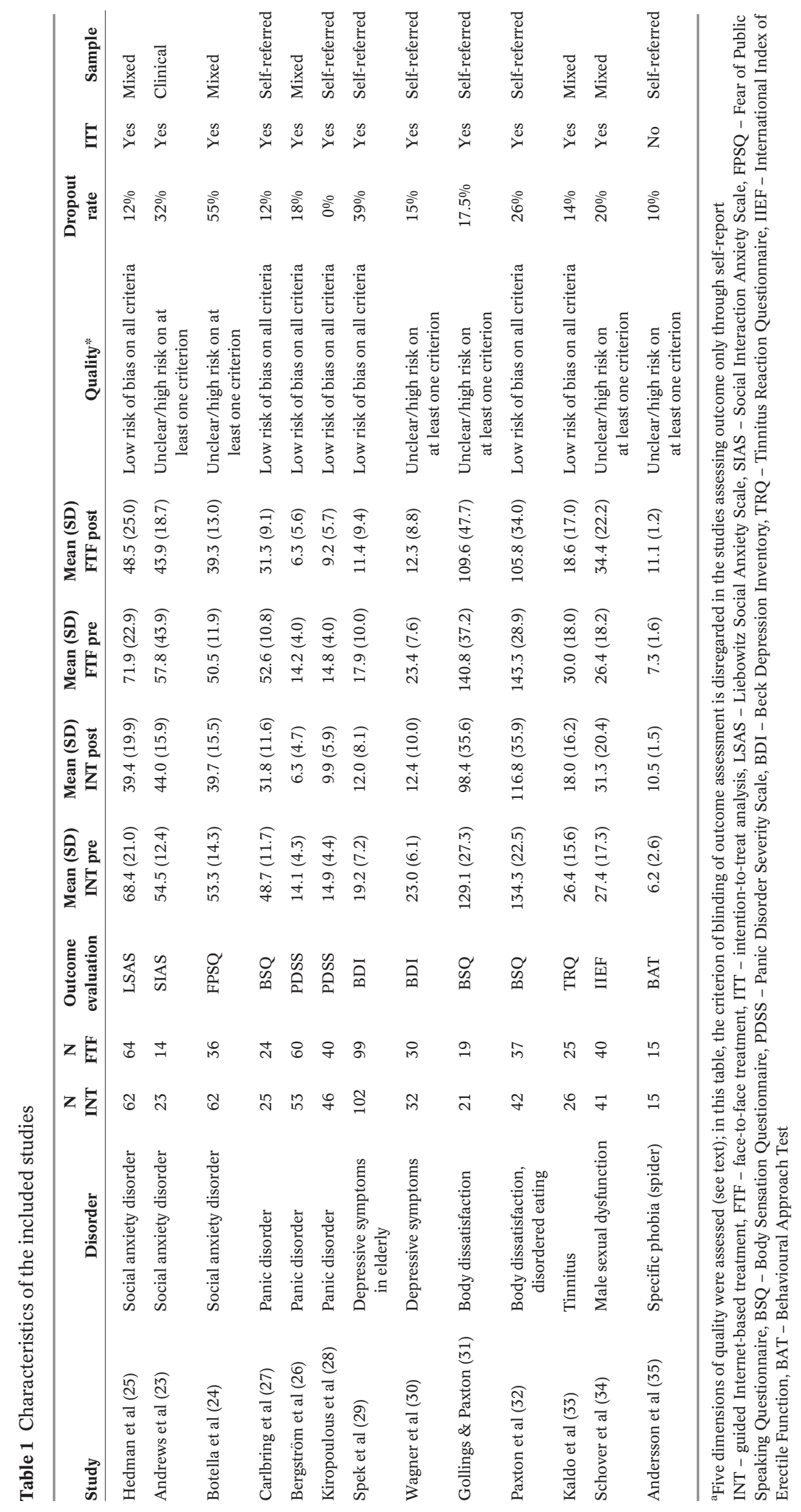




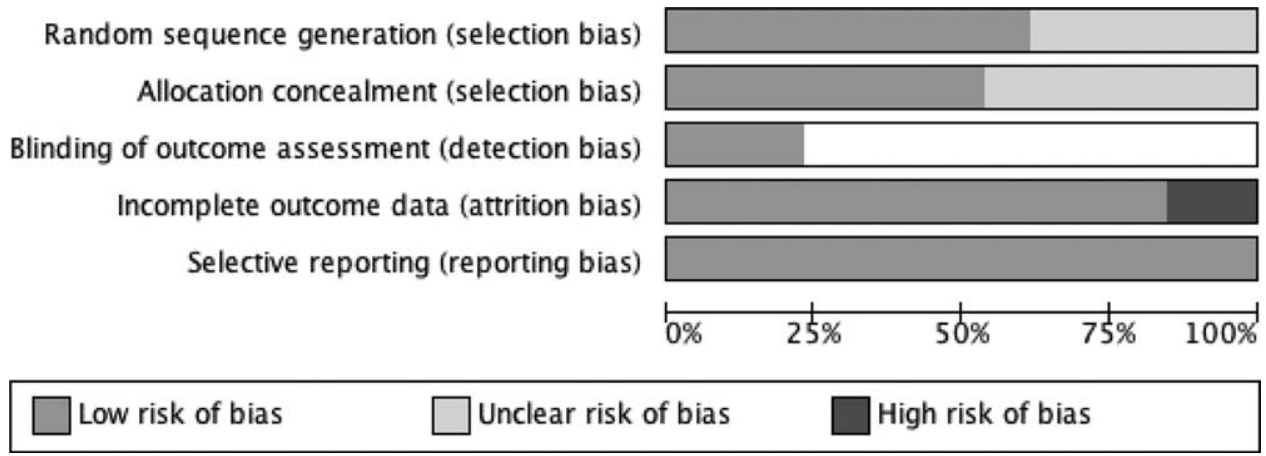

Figure 2 Estimated risk of bias across all included studies

did not search for unpublished studies. There were no restrictions regarding publication date. Searches were last updated in July 2013. We also consulted other databases (Scopus, Google Scholar and PsychInfo), and reference lists of recent studies and reviews on Internet interventions.

Two researchers read the abstracts independently and, in case of disagreement on inclusion, they discussed it amongst themselves or asked a third researcher for advice.

Each included study was assessed for quality using the criteria proposed by the Cochrane collaboration (20). Five dimensions were assessed: risk of selection bias due to the method for generating the randomization sequence; risk of selection bias in terms of allocation concealment, i.e., due to foreknowledge of the forthcoming allocations; detection bias in terms of blinding of outcome assessors; attrition bias due to incomplete outcome data; and reporting bias due to selective reporting of results. The criterion for performance bias relating to masking of participants was not used, as that form of masking is not possible in the types of treatments investigated in this review. On each dimension, the status of the studies was rated using the response options "low risk", "high risk" or "unclear". The alternative "unclear" was used when there was no data to assess the quality criterion in the original study. In studies using self-report, the criterion of blinding of outcome assessors was judged to be not applicable.

Data were analysed using Review Manager (RevMan) version 5.1.0 (20). In the main meta-analyses, we assessed the effect of guided ICBT compared to face-to-face treatment using the standardized mean difference at post-treatment (Hedges' g) as outcome, meaning that the difference between treatments was divided by the pooled standard deviation. If both intention-to-treat and per-protocol data were presented, the former estimate was used in the meta-analysis. Estimates of treatment effects were conducted both using all included studies and separately for each clinical disorder (e.g., depression). Potential differences in dropout rates between guided ICBT and face-to-face treatment were analysed using meta-analytic logistic regression.

All pooled analyses were carried out within a random effects model framework, assuming variation in true effects

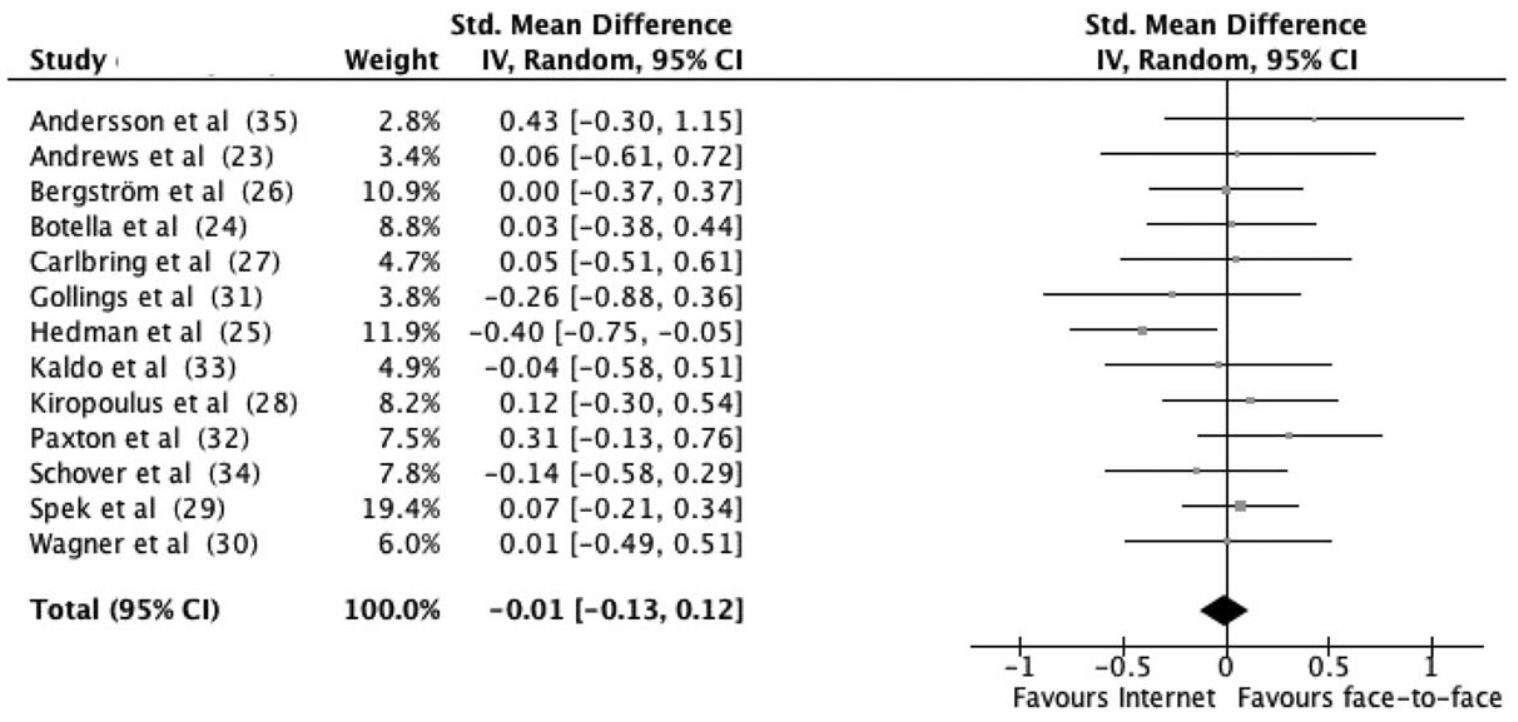

Figure 3 Forest plot displaying effect sizes of studies comparing guided Internet-based treatment with face-to-face treatment 


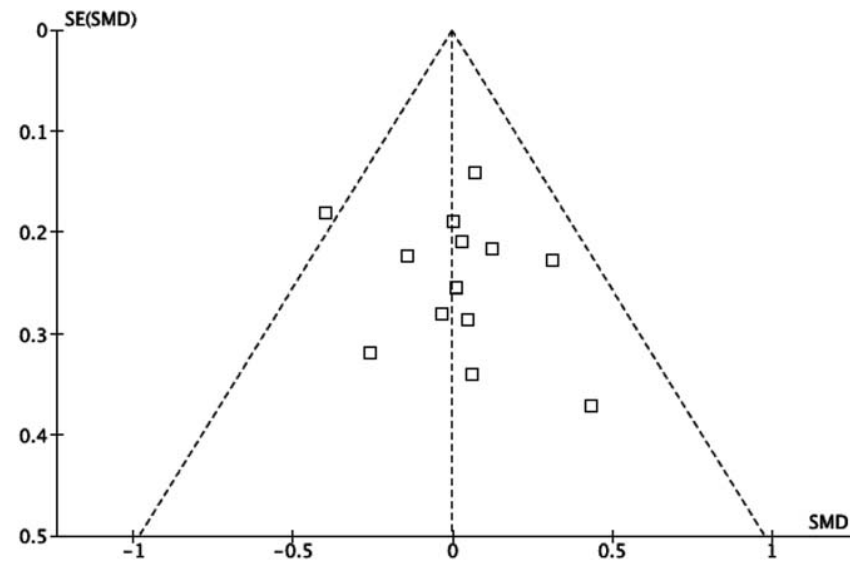

Figure 4 Funnel plot to assess for publication bias by relating effect sizes of the studies to standard errors. SE - standard error, SMD standardized mean difference (Cohen's d)

in the included studies and accounting for the hypothesized distribution of effects $(21,22)$. Studies were assessed for heterogeneity using $\chi^{2}$ and $\mathrm{I}^{2}$ tests, where an estimate above $40 \%$ on the latter test suggests presence of heterogeneity (21). In addition, forest plots were inspected to assess variation in effects across studies. Sensitivity analyses were conducted to assess whether study quality was related to outcome, by comparing studies judged as having a low risk of bias on all five quality criteria dimensions with the other studies (i.e., those assessed as "unclear" or "high risk" on at least one quality criterion). Publication bias was investigated using funnel plots.

Power calculations were conducted as suggested by Borenstein et al (22) and showed that, in order to have a power of $80 \%$ to detect a small effect size $(\mathrm{d}=0.3)$, given an alpha-level of $0.05,14$ studies with an average of 25 participants in each treatment arm were needed.

\section{RESULTS}

Of 1,286 screened studies, 13 (total $\mathrm{N}=1053$ ) met all review criteria and were included in the analysis. Figure 1 displays the study inclusion process. All the 13 studies investigated guided ICBT against some form of CBT (individual format, $n=6$ and group format, $n=7$ ). In terms of conditions studied, three targeted social anxiety disorder (23-25), three panic disorder (26-28), two depressive symptoms $(29,30)$, two body dissatisfaction $(31,32)$, one tinnitus (33), one male sexual dysfunction (34), and one spider phobia (35). The total number of participants was 551 in guided ICBT and 502 in the face-to-face condition.

The studies were conducted by eight independent research groups and carried out in Australia, the Netherlands, Spain, Sweden, Switzerland, or the U.S.. The smallest study had 30 participants and the largest 201. Seven studies recruited participants solely through self-referral, while the remainder included participants from clinical samples or using a mix of self-referral and clinical recruitment. All studies were published between 2005 and 2013. The characteristics of each study are presented in Table 1.

When blinding of outcome assessment was included, only three studies were judged as having low risk of bias on all five quality dimensions $(25,26,28)$. When that criterion was disregarded in the studies assessing outcome only through self-report, 7 of 13 studies were judged as having low risk of bias on all quality dimensions. Figure 2 displays the averaged risk of bias in the included studies.

In terms of dropout, meta-analytic logistic regression showed no significant difference between the two treatment formats $(\mathrm{OR}=0.79 ; 95 \% \mathrm{CI}$ : 0.57-1.09), indicating that dropout did not systematically favour one treatment over the other. Tests of heterogeneity did not demonstrate significant differences in effects across treatments $\left(\chi^{2}=9.91 ; \mathrm{I}^{2}=\right.$ $0 \% ; \mathrm{p}=0.62)$.

A forest plot presenting effect sizes ( $\mathrm{g}$ ) of each study as well as the pooled between-group effect size of all studies is presented in Figure 3. An effect size estimate below 0 favours guided ICBT, while an effect size above 0 represents larger effects for face-to-face CBT. The pooled betweengroup effect size (g) at post-treatment across all 13 studies was -0.01 (95\% CI: -0.13 to 0.12$)$, showing that guided ICBT and face-to-face treatment produced equivalent overall effects.

In the three studies targeting social anxiety disorder (23$25)$, the pooled between-group effect size (g) was -0.16 (95\% CI: -0.47 to 0.16 ), in favour of guided ICBT but indicating equivalent effects. In the three studies targeting panic disorder (26-28), the effect size was 0.05 (95\% CI: -0.20 to 0.30 ), in line with the notion of equivalent effects. In the two studies targeting depressive symptoms $(29,30)$, the effect size was 0.05 (95\% CI: -0.19 to 0.30 ), showing equivalent effects for this condition as well.

In the two studies targeting body dissatisfaction $(31,32)$, the effect size was 0.07 (95\% CI: -0.49 to 0.62$)$, again showing largely equivalent effects. In the only study targeting tinnitus (36), the effect size was -0.04 (95\% CI: -0.58 to 0.51 ), suggesting no difference between the formats for this condition as well. In the only study targeting male sexual dysfunction (34), using a clinical sample of patients that had been treated for prostate cancer, the effect size was -0.14 (95\% CI: -0.58 to 0.29 ), which is a small effect again in slight favour of ICBT. In the only study targeting spider phobia (35), the effect size was 0.43 (95\% CI: -0.30 to 1.15 ), in favour of face-to-face treatment, but given the small size of the study not significant.

In order to estimate whether there was an association of study quality and treatment effects, subgroup analyses were conducted. In the three studies judged to have low risk of bias on all five quality criteria, the pooled effect size (g) was -0.11 (95\% CI: -0.42 to 0.21 ), while it was 0.05 (95\% CI: -0.10 to 0.19 ) for the other ten studies, suggesting that study quality did not affect outcomes significantly. 
Figure 4 presents a funnel plot relating effect sizes on the primary outcome of the studies to the standard errors of the estimates. Effect sizes were evenly distributed around the averaged effect. Of specific interest, the lower right section of the funnel plot is not devoid of studies, suggesting that there is no major bias of the pooled effect estimate due to unpublished small studies with results favouring face-toface treatment.

\section{DISCUSSION}

The aim of this systematic review and meta-analysis was to collect and analyse studies in which guided ICBT had been directly compared with face-to-face CBT. Altogether, the findings are clear in that the overall effect for the main outcomes was close to zero, indicating that the two treatment formats are equally effective in social anxiety disorder, panic disorder, depressive symptoms, body dissatisfaction, tinnitus, male sexual dysfunction, and spider phobia, when analysed as an aggregated cohort.

Thus, the present meta-analytic review mirrors the findings by Cuijpers et al (18), who found no differences between guided self-help and face-to-face therapies. Interestingly, there is only a minor overlap between that metaanalysis and the present one. We included the studies by Spek et al (29) and Botella et al (24), as they involved therapist contact in association with inclusion (but not during treatment). We did not include a study (included in Cuijpers et al's meta-analysis) that was judged to compare two forms of ICBT rather than ICBT vs. face-to-face treatment (37).

While there were relatively few studies on each condition, the overall number of studies and number of participants gave us power to detect differences of importance between the formats. There was a low risk of bias, including publication bias, but many individual studies were much underpowered to detect differences, and for each of the included conditions there were few studies and sometimes only one.

The results of this meta-analysis are thought-provoking both from a theoretical and practical point of view. In terms of theories about change in psychotherapeutic interventions, the results suggest that the role of a face-to-face therapist may not be as crucial as suggested in the literature (38) to generate large treatment effects. Even if factors such as therapeutic alliance are established in guided ICBT (39), they are rarely important for outcome. Indeed, understanding what makes ICBT work is a challenge for future research, as only a few studies to date have investigated mediators of outcome (e.g., 40,41).

From a practical point of view, the findings call for research on treatment preferences and effectiveness in real life settings, as most studies in this review involved selfreferred participants recruited via advertisements. There are studies on treatment acceptability of ICBT showing that patient tend to appreciate the ICBT format (42-44), but also one study reporting the opposite (45). When it comes to effectiveness, there are now at least four controlled trials and eight open studies showing that ICBT works in regular clinical settings (46). However, controlled trials such as the ones reviewed in this meta-analysis all require that participants consent to being randomized to either ICBT or face-to-face treatment, a requirement that limits the generalizability of the results.

The present meta-analysis has several strengths, such as a consistent outcome across studies regarding efficacy of guided ICBT compared to face-to-face CBT, the relatively high quality of the trials included, little heterogeneity and no indication of publication bias. However, there are also limitations. First, the included studies differed substantially in terms of treatment content, not so much within studies as between ICBT programs. We endorsed a broad definition of CBT, but it would of course have been preferable to have many studies on the same program, as is the case in reviews of cognitive therapy for depression (47). Second, we compared against different formats of face-to-face therapy and it could be argued that group CBT is a suboptimal comparison (48), at least when it comes to patient preferences. Third, we analysed the primary outcome measures in the trials and did not include secondary outcomes. Indeed, the heterogeneity of clinical conditions included can be viewed as a problem on its own, but we cannot at this stage and with very few studies for each condition conclude that guided ICBT and face-to-face therapy are equally effective on all outcomes. For example, there are very few studies on knowledge acquisition following CBT and even fewer on ICBT (49), and this can be something that differs between the therapy formats (in particular in the long run). In addition, patient characteristics have not been taken into account. This is potentially important, since there are studies suggesting that different predictors of outcome (e.g., agoraphobic avoidance) are relevant when comparing face-to-face versus Internet treatment. Fourth, we only included studies on adult samples. However, a study by Spence et al (50) on adolescents is clearly in line with our findings, suggesting equivalence. Finally, we did not analyse long-term effects of the treatments. This is a possible area for future research, as the type of trials included here has the advantage that randomization can be maintained for long time periods.

ICBT has only been around for a short time and is still developing rapidly (51). A recent change is the use of mobile smart phones in treatment, and it is likely that smart phone applications and ICBT will blend in with face-to-face treatment in the near future. Finally, while we performed this review in the form of a study-level meta-analysis, there is an emerging trend to instead conduct patient-level meta-analyses with primary data (52).

In conclusion, guided ICBT has the promise to be an effective, and potentially cost-effective, alternative and complement to face-to-face therapy. More studies are needed before firm conclusions can be drawn, but the findings to date, including this meta-analysis, clearly show that guided ICBT is a treatment for the future. 


\section{Acknowledgements}

G. Andersson acknowledges Linköping University, the Swedish Science Foundation and Forte for financial support.

\section{References}

1. Andersson G, Carlbring P, Ljótsson B et al. Guided Internetbased CBT for common mental disorders. J Contemp Psychother 2013;43:223-33.

2. Hedman E, Ljótsson B, Lindefors N. Cognitive behavior therapy via the Internet: a systematic review of applications, clinical efficacy and cost-effectiveness. Expert Rev Pharmacoecon Outcomes Res 2012;12:745-64.

3. Titov N, Dear BF, Johnston L et al. Improving adherence and clinical outcomes in self-guided internet treatment for anxiety and depression: randomised controlled trial. PLoS One 2013;8: e62873.

4. Andersson G, Carlbring P, Berger T et al. What makes Internet therapy work? Cogn Behav Ther 2009;38(S1):55-60.

5. Lange A, Rietdijk D, Hudcovicova M et al. Interapy: a controlled randomized trial of the standardized treatment of posttraumatic stress through the Internet. J Consult Clin Psychol 2003;71:901-9.

6. Kessler D, Lewis G, Kaur S et al. Therapist-delivered internet psychotherapy for depression in primary care: a randomised controlled trial. Lancet 2009;374:628-34.

7. Johansson R, Andersson G. Internet-based psychological treatments for depression. Expert Rev Neurother 2012;12:861-70.

8. Andersson G. Using the internet to provide cognitive behaviour therapy. Behav Res Ther 2009;47:175-80.

9. Johansson R, Ekbladh S, Hebert A et al. Psychodynamic guided self-help for adult depression through the Internet: a randomised controlled trial. PLoS One 2012;7:e38021.

10. Ström M, Uckelstam C-J, Andersson G et al. Internet-delivered therapist-guided physical activity for mild to moderate depression: a randomized controlled trial. PeerJ 2013;1:e178.

11. Carlbring P, Apelstrand M, Sehlin $\mathrm{H}$ et al. Internet-delivered attention training in individuals with social anxiety disorder - a double blind randomized controlled trial. BMC Psychiatry 2012;12:66.

12. Warmerdam L, van Straten A, Twisk J et al. Internet-based treatment for adults with depressive symptoms: randomized controlled trial. J Med Internet Res 2008;10:e44.

13. Buhrman M, Skoglund A, Husell J et al. Guided Internet-delivered acceptance and commitment therapy for chronic pain patients: a randomized controlled trial. Behav Res Ther 2013;51:307-15.

14. Andersson G, Cuijpers P. Internet-based and other computerized psychological treatments for adult depression: a meta-analysis. Cogn Behav Ther 2009;38:196-205.

15. Andrews G, Cuijpers P, Craske MG et al. Computer therapy for the anxiety and depressive disorders is effective, acceptable and practical health care: a meta-analysis. PLoS One 2010;5:e13196.

16. Cuijpers P, van Straten A-M, Andersson G. Internet-administered cognitive behavior therapy for health problems: a systematic review. J Behav Med 2008;31:169-77.

17. Hedman E, Furmark T, Carlbring P et al. Five-year follow-up of internet-based cognitive behaviour therapy for social anxiety disorder. J Med Internet Res 2011;13:e39.

18. Cuijpers P, Donker T, van Straten A et al. Is guided self-help as effective as face-to-face psychotherapy for depression and anxiety disorders? A meta-analysis of comparative outcome studies. Psychol Med 2010;40:1943-57.

19. Thomson AB, Page LA. Psychotherapies for hypochondriasis. Cochrane Database Syst Rev 2007(4):CD006520.
20. Higgins J, Green S (eds). Cochrane handbook for systematic reviews of interventions. Version 5.1.0. The Cochrane Collaboration, 2011.

21. Crowther M, Lim W, Crowther MA. Systematic review and metaanalysis methodology. Blood 2010;116:3140-6.

22. Borenstein M, Hedges LV, Higgins JPT et al. Introduction to meta-analysis. Chichester: Wiley, 2009.

23. Andrews G, Davies M, Titov N. Effectiveness randomized controlled trial of face to face versus Internet cognitive behaviour therapy for social phobia. Aust N Z J Psychiatry 2011;45:337-40.

24. Botella C, Gallego MJ, Garcia-Palacios A et al. An Internet-based self-help treatment for fear of public speaking: a controlled trial. Cyberpsychol Behav Soc Netw 2010;13:407-21.

25. Hedman E, Andersson G, Ljótsson B et al. Internet-based cognitive behavior therapy vs. cognitive behavioral group therapy for social anxiety disorder: a randomized controlled non-inferiority trial. PLoS One 2011;6:e18001.

26. Bergström J, Andersson G, Ljótsson B et al. Internet-versus group-administered cognitive behaviour therapy for panic disorder in a psychiatric setting: a randomised trial. BMC Psychiatry 2010;10:54.

27. Carlbring P, Nilsson-Ihrfelt E, Waara J et al. Treatment of panic disorder: live therapy vs. self-help via Internet. Behav Res Ther 2005;43:1321-33.

28. Kiropoulos LA, Klein B, Austin DW et al. Is internet-based CBT for panic disorder and agoraphobia as effective as face-to-face CBT? J Anxiety Disord 2008;22:1273-84.

29. Spek V, Nyklicek I, Smits $N$ et al. Internet-based cognitive behavioural therapy for subthreshold depression in people over 50 years old: a randomized controlled clinical trial. Psychol Med 2007;37:1797-806.

30. Wagner B, Horn AB, Maercker A. Internet-based versus face-toface cognitive-behavioral intervention for depression: a randomized controlled non-inferiority trial. J Affect Disord 2014;152154:113-21.

31. Gollings EK, Paxton SJ. Comparison of internet and face-to-face delivery of a group body image and disordered eating intervention for women: a pilot study. Eat Disord 2006;14:1-15.

32. Paxton SJ, McLean SA, Gollings EK et al. Comparison of face-toface and internet interventions for body image and eating problems in adult women: an RCT. Int J Eat Disord 2007;40:692-704.

33. Kaldo V, Levin S, Widarsson $\mathrm{J}$ et al. Internet versus group cognitive-behavioral treatment of distress associated with tinnitus. A randomised controlled trial. Behav Ther 2008;39:348-59.

34. Schover LR, Canada AL, Yuan Y et al. A randomized trial of internet-based versus traditional sexual counseling for couples after localized prostate cancer treatment. Cancer 2012;118:500-9.

35. Andersson G, Waara J, Jonsson U et al. Internet-based self-help vs. one-session exposure in the treatment of spider phobia: a randomized controlled trial. Cogn Behav Ther 2009;38:114-20.

36. Kaldo V, Levin S, Widarsson J et al. Internet versus group cognitive-behavioral treatment of distress associated with tinnitus: a randomized controlled trial. Behav Ther 2008;39:348-59.

37. Tillfors M, Carlbring P, Furmark T et al. Treating university students with social phobia and public speaking fears: Internet delivered self-help with or without live group exposure sessions. Depress Anxiety 2008;25:708-17.

38. Wampold BE. The great psychotherapy debate. Models, methods, and findings. Mahaw: Lawrence Erlbaum, 2001.

39. Andersson G, Paxling B, Wiwe $M$ et al. Therapeutic alliance in guided Internet-delivered cognitive behavioral treatment of depression, generalized anxiety disorder and social anxiety disorder. Behav Res Ther 2012;50:544-50.

40. Hedman E, Andersson E, Andersson G et al. Mediators in Internet-based cognitive behavior therapy for severe health anxiety. PLoS One 2013;8:e77752. 
41. Hesser H, Zetterqvist Westin V, Andersson G. Acceptance as mediator in Internet-delivered acceptance and commitment therapy and cognitive behavior therapy for tinnitus. J Behav Med 2014;37:756-67.

42. Wootton BM, Titov N, Dear BF et al. The acceptability of Internet-based treatment and characteristics of an adult sample with obsessive compulsive disorder: an Internet survey. PLoS One 2011;6:e20548.

43. Spence J, Titov N, Solley K et al. Characteristics and treatment preferences of people with symptoms of posttraumatic stress disorder: an internet survey. PLoS One 2011;6:e21864.

44. Gun SY, Titov N, Andrews G. Acceptability of Internet treatment of anxiety and depression. Australas Psychiatry 2011;19:259-64.

45. Mohr DC, Siddique J, Ho J et al. Interest in behavioral and psychological treatments delivered face-to-face, by telephone, and by internet. Ann Behav Med 2010;40:89-98.

46. Andersson G, Hedman E. Effectiveness of guided Internetdelivered cognitive behaviour therapy in regular clinical settings. Verhaltenstherapie 2013;23:140-8.
47. Cuijpers P, Berking M, Andersson G et al. A meta-analysis of cognitive behavior therapy for adult depression, alone and in comparison to other treatments. Can J Psychiatry 2013;58:376-85.

48. Morrison N. Group cognitive therapy: treatment of choice or suboptimal option? Behav Cogn Psychother 2001;29:311-32.

49. Andersson G, Carlbring P, Furmark T et al. Therapist experience and knowledge acquisition in Internet-delivered CBT for social anxiety disorder: a randomized controlled trial. PloS One 2012;7:e37411.

50. Spence SH, Donovan CL, March S et al. A randomized controlled trial of online versus clinic-based CBT for adolescent anxiety. J Consult Clin Psychol 2011;79:629-42.

51. Andersson G, Titov N. Advantages and limitations of Internetbased interventions for common mental disorders. World Psychiatry 2014;13:4-11.

52. Bower P, Kontopantelis E, Sutton AP et al. Influence of initial severity of depression on effectiveness of low intensity interventions: meta-analysis of individual patient data. BMJ 2013;346:f540.

DOI 10.1002/wps.20151 